\title{
Study on Ai Performance and Possibilities for Future Wars
}

\author{
Naveen Rathi
}

\begin{abstract}
Objective: Too much reliance is being laid on applications of AI in finding solution to various problems being faced by human kinds. The recent outbreak of COVID 19 is comparable to outbreak of any other havoc on the mankind including war, due to similar characteristics of three Vs i.e. Velocity; Volume and Veracity. The objective of study is to draw out lessons from AI applications and success achieved during pandemic spread for the application of AI in future wars. Method : The study has been done in two parts first part consist of descriptive analysis of performance of $A I$ in predicting, diagnosing and finding the resolution to current pandemic. The second part consists of working out the way forward for application of AI during future warfare. The methodology used for working out expected Ai performance during future warfare has been survey and interaction with the experts in the field. Findings :The findings of the study have been that AI has been quite a success story during prediction and diagnosis stage of Pandemic but the actual performance in finding solution to the problem has been questionable. As a result the countries world over have suffered huge losses and had to resort to various preventions measures. Novelty: The novelty of the study is derived from the fact that various study in the AI field have been specific to a particular field and no work was done on learning the lessons from one domain to its application to other field of similar nature. The question of "How much reliance should be laid upon AI during a future war." has largely remained unanswered. With reference to ethical and legal issues related to AI in employment of Killer Robots the study recommends future course of action. We need to ensure that no rogue application of AI takes place in a sector where the very existence of humanity is at stake.

Keywords: Artificial Intelligence (AI), Lethal Automated
\end{abstract} weapon system (LAWS), Killer Robots, Ethical Governors

\section{INTRODUCTION}

1. With advancement of technology we have not only got digitised but also digitalised which sets the space for rapid induction of newer technologies like AI, Machine learning, Virtual reality, Internet of Things, Big Data Analytics. These technologies influence all walks of life and also the way future wars would be fought. The prime focus of AI was to build machines that are able to perform tasks which require intelligence, such as reasoning, learning, planning, problem solving and perception as well as the ability to detect, classify and respond to the user's emotions and other stimuli [1]. The task during war without any human interference using only

Revised Manuscript Received on October 10, 2020.

* Correspondence Author

Mr. Naveen Rathi*, Post Graduate of Weapon Technology and Done PGDM in Finance.

(c) The Authors. Published by Blue Eyes Intelligence Engineering and Sciences Publication (BEIESP). This is an open access article under the CC BY-NC-ND license (http://creativecommons.org/licenses/by-nc-nd/4.0/) thought of an AI machine to have the ability to perform any

the computer programming and algorithms has become a reality now.

The problem of big data analytics of three $\mathbf{V}$ 's i.e. Larger Volume, Velocity of data flow and Variety of data during future conflicts was experienced during recent outbreak of COVID 19. The volume of infected cases and velocity with which $R_{0}$ changed, the large volumes of active cases are comparable with data generated during war in a 24 hr cycle at Division level which is about 1-2 TBs and at Corps level five times of it. If a Strike Corps plans its operations after four days, the volume of data required to be analysed would be more than 50,000 GBs/0.05 Petabytes. However, in the physical domain only $10 \%$ would be analysed. The varied behaviour of virus to different modus operandi of handling the situation and varied results achieved is similar to different war scenario each time [2].

\section{STUDY OBJECTIVES}

The prediction of pandemic from Wuhan along with likely spread to other countries was initially based on the flight data of various flights from China. However, for last six months the world is striving hard to diagnose the spread, limit it and finally get the solution to the problem. In absence of any technical solution a majority of the countries had to resort to Lockdown - a 100 yrs old method to control Spanish flu. Does it imply that more than real expectations were put on AI world over and if such is the result, the employment of AI machines (Killer Robots) during war needs to be done with care. Whether the Killer Robots of future would be intelligent enough to avoid collateral damage or result in situation going out of control, which may be due to a software glitch or break in communication. The objective of study is to draw out lessons from AI applications and success achieved during pandemic spread for the future wars.

\subsection{Research Question}

In the backdrop of AI employment during COVID 19, in this research, through the literature study and survey method, we have attempted to find answers to the following questions:2.1.1 What has been the AI employment during handling of COVID19 crisis?

2.1.2 What would be the likely areas of application and effect of employment of AI machines in future wars?

2.1.3 Ethical issues while employing Lethal Autonomous Weapon System (LAWS) during war.

\section{RESEARCH METHODOLOGY}

3.1 Sample Size and Sources of Data - In this study primary data was collected through interviews by using questionnaire. The questionnaire was filled by 123 scholars from defense sector and academia to include planning and execution level officers of different service experience.

Blue Eyes Intelligence Engineering \& Sciences Publication

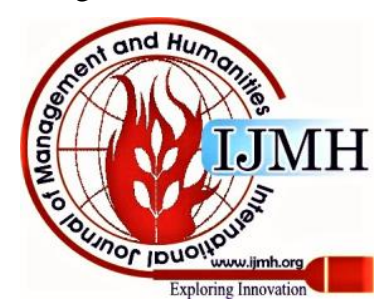




\section{Study on Ai Performance and Possibilities for Future Wars}

The secondary data was collected from books, websites, research reports and journals.

3.2 Tools of Study - In the study we used descriptive statistics like mean, Standard deviation and variance to analyse the data. The results found are represented in the form of pie charts in the appendix.

\subsection{Limitation of the Study}

3.3.1 This is subjected to current situation and prejudices of the respondents; hence $100 \%$ accuracy cannot be assured.

3.3.2 The research was carried out in a short span of time, where in the research could not widen the study.

\section{APPLICATIONS OF AI DURING COVID19}

For finding the solution to any problem including pandemic and war like situation AI can be employed at three stages i.e. for Prediction, Detection and Solution. Executive Director of the communicable disease cluster, WHO, David Haymen explains that AI or no AI several key factors are required for successful public health response to an outbreak of a new infection. These factors include understanding of transmissibility, and risk population; establishing the natural history of infection, including incubation period and mortality rate; identifying and characterising the causative organism and in some instances epidemiological modelling to suggest effective prevention and control measures [3]. All these factors require collection and analysis of data in real time frame, which was made possible due to spread of internet and software applications. Since last historical data of spread of global pandemic dates back to Spanish Flu 1918 the authenticity is limited. Few areas where AI has found significant application during pandemic are as follows [4]:-

4.1 Prediction of Problem - It has been claimed that various companies found AI to be useful in identifying likely hotspots. On 30 Dec 19 Blue Dot, a start-up AI company of Canada had predicted spread of an endemic by seeing an unusual rise in pneumonia cases in Wuhan, China. The issue which was flagged nine days later by World Health Organisation [5].

4.2 Detect Infection with Speed and Accuracy - The spread of disease could be predicted using the flight data from Wuhan. AI is helpful in diagnosing infected patients with help of medical imaging technologies by human scan in faster time frame. Infervision, a Beijing based AI company uses its algorithm to spot COVID 19 on images of the lung as distinct from the other respiratory infections. The AI system from Alibaba is supposed to train 5000 CT scans of corona virus patients claims 96\% accuracy in differentiating cases of COVID 19 and pneumonia viruses [6]. Baidu has claimed to develop a tool called linera fold which slashed the time taken in detection of Corona virus from 55 minutes to 27 seconds [7].

4.3 Monitoring the Situation - Day to day update of patients can be monitored with help of AI. From contactless monitoring of situation and reaction to administered drugs the AI is presently involved in all phases of monitoring. As reported by New York Times for identification of Corona infected person China is currently using 200 million surveillance cameras. In various municipalities the combination of facial recognition technology and AI is being used to the benefit of authorities to identify COVID19 suspects [8].

4.4 Reducing Human Load - With early diagnosis and providing treatment at an early stage using digital approach;
AI has reduced the workload on health workers considerably in conduct of routine tests and serving patients.

4.5 Projection of cases and Mortality - With the availability of data the projections and mathematical models could be developed to forecast No of infected persons and likely deaths. Different projections are made for a country with weaker health system or time taken by the government in taking decision. The number of confirmed cases and death in particular region based on available data could be predicted [9].

4.6 Finding a Solution Vaccine - AI is currently being used for speeding up the development of drugs and conduct of clinical trials. With the help of real time analysis AI can provide updated information on effect of drug on human body.

\section{AI APPLICATIONS DURING FUTURE WARS}

Brencht (1930) penned a poem "general here we have a handy little gadget / called a 'man'; he can fly a plane / he can kill. He has only one defect / he can think." Well some think that robot of future won't suffer that defect which is why were frightened of them because they won't be able to think through their actions. others fear that they will acquire the ability to think for themselves and unlike us they won't be so easy to socialise into obedience[10]. AI has the potential of "dual-use" technology both for military and civil purposes. Self-driving cars rely on programmed route and traffic conditions using array of sensors. The same technology and reconfigured versions of the same algorithms will one day be applied to self-driving tanks set loose on future battlefields [11]. Few major applications in defense where AI has a ground-breaking role to play are:-

\subsection{Prediction due to Improved Transparency}

Similar to early detection and prediction during COVID19 the AI applications during war for Intelligence, Surveillance, and Reconnaissance has two domains, first a system of sensors i.e. unmanned vehicles, ground based sensors to collect data and second is analysis of collected data for extraction of intelligence. Opposing forces would use swarms of sensors like drones for surveillance. US military AI systems named 'Project Maven' incorporates machine vision and computer learning algorithms into intelligence collection cells that combined through footage from RPAS automatically identifies hostile activity for targeting [12]. On line of Killer robots, Information Robots would soon be developed to move unhindered during operations and create communication network which would be mobile and flexible. It would facilitate comprehensive situational awareness for senior commanders for timely and optimum decision making. 5.2 Detect and Engage Targets with Speed and Accuracy Researchers at the University of Manchester, UK and University of Madrid, Spain have developed an AI based system that could identify people from their gait and walking pattern with a success rate of $99.3 \%$. Such a system could be employed for surgical strike on enemy command and control elements [13]. Through satellite based control links, UCAVs can now be remotely manoeuvred over any part of the globe and can take over the functions of manned fighter aircrafts in future. America's semi-autonomous long range anti-ship missile may need human to identify targets but it deploys AI to decide how to destroy them.

Published By:

Blue Eyes Intelligence Engineering \& Sciences Publication

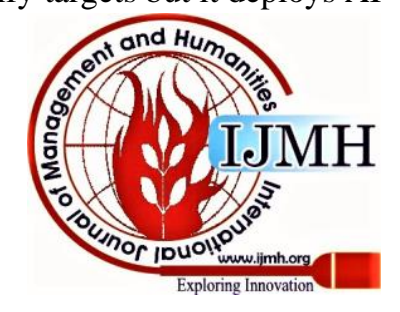


Israel has developed loitering missile Harop; which loiters in the sky for considerable period until a target appears. The American anti submarine vessel, Sea hunter can cruise the ocean for months at a time scouting for enemies with no one abroad.

\subsection{Reduce Human Interference in Logistics}

AI would replace all push/ pull model of providing logistics in right time, right place and right conditions. The real time and projected monitoring of logistics expenditure by troops in combat would result in automated placement of orders for replenishments to the Supply depot. Recording of move forward of logistics with the help of barcoding and RFID would make entire procedure more efficient and accurate.

\subsection{Monitor Mental Health}

Conditions in war would mandate an increasing number of cases with emotional and behavioural disorders in armed forces. With the help of AI these personals would get efficient treatment from health care professionals. AI in healthcare industry is set to become a $\$ 36.1 \mathrm{Bn}$ market by 2025 [14]. Several countries are looking forward to develop a complete AI enabled systems to track objects, provide real-time intelligence and minimise causalities during future operations.

\section{CHALLENGES FOR APPLICATION OF AI}

6.1 As Paul Scharre (2019) puts it in his book Army of None, "Humans are not perfect, but they can empathize with their opponents and see the bigger picture. Unlike humans, autonomous weapons would have no ability to understand the consequences of their actions, no ability to step back from the brink of war." [15]. The important short comings of machine are:-

6.1.1 Machines are intelligent but directionless. Humans have motivated intelligence, mix of emotions, instructs, instincts and drive programmed into them. Machines do not have moral standing, Robots do not choose whether to follow their programme or not; consistency is their strong suit.

6.1.2 Machines are non-teleological. Human think with reason whereas machines are based on logic. What am I doing on the battlefield? Why am I taking risks? Are not the questions which occur to an AI Machine. Machines may be programmed to self-destruct but for them it is not martyrdom and there is no emotional register.

6.2 With above technologies in place the future scenario would encompass Robotic vehicles who will accompany troops on the battlefield, carrying supplies and firing on enemy positions; swarms of armed drones will attack enemy tanks, radars, and command centre; unmanned under sea vehicles, will pursue both enemy submarines and surface ships. At the outset of combat, all these LAWS will be controlled by humans. However, as the fighting would intensify the communications between headquarters and the front lines may be lost and such systems will be on their own, empowered to take lethal action without further human intervention [16].

6.3 This kind of scenario throws various challenges at defense planners. The challenges required to be answered by defense planners are:-

6.3.1 Unstructured Data - 'Cognitive' machines learn from their interactions with humans and the environment . They are probabilistic, that is they provide not answers to numerical problems but confidence-weighted responses together with supporting evidence. They consistently identify patterns and provide fresh insights while analysing unstructured data [17]. Such data might not be available during war given more than 80 per cent of the data in war would be unstructured. Hence each machine would behave differently. The reason quoted for AI not being much successful during COVID 19 is non availability of structured data or historic data.

6.3.2 Defence vs Civil Applications - Driverless car Development originated with a Defense Advanced Research Projects Agency (DARPA) programme in 2004. When the programme ended in 2007, driverless cars could move only slowly through closed courses, and not without accidents. On commercial front a decade later, Industry is on the verge of commercialising driverless cars around the world [18]. It goes to confirm that development in AI would continue in civil sector and is unstoppable now.

6.3.3 Bugs in the Machine - Possibility of rogue AI development in future by non-state actors cannot be denied or is already on. The US defense think tank Rand Corporation had warned in a study that the use of AI in military applications could give a rise to a nuclear war by 2040. The worries arose from the infamous incident of 1983, when former soviet military officer Stanisalav Petrov noticed that Russian computers had incorrectly put out a warning that the US had launched nuclear missiles, averting nuclear war [19]. In short, Algorithms are neither secure and nor they are immune to bugs, malware, bias and manipulation. Such was the impact felt during development of vaccine where all countries put high claims that they were on the verge of developing a vaccine.

6.3.4 Reduced Human Space - We must not fall prey to the mistaken notion that technology can reduce warfare to simply manning the machines. LAWS have been classified into three categories. Human in the Loop; LAWS can select target, while human takes kill decision; Human on the Loop weapons can select and take kill decision autonomously, while a human can override the decision by exerting oversight ; and Human out of the Loop LAWS are those that may select and engage targets without any human interaction [20]. Now within these three categories human control has to be exercised. Introducing Human in the OODA loop becomes the weakest link and hence most vulnerable. At the same time it would slow the entire OODA loop thus loosing the vary advantage of AI. As a white paper from the US Army Training and Doctrine Command (TRADOC) states that autonomous unmanned system will be fully adaptive to unforeseen changes while remaining completely predictable in mission performance . Quoting from the paper "Advances in computer architecture and Machine intelligence will have reached a point where intelligent agents can analyse the environment and current battle situation , search likely target areas, detect and analyse targets, assist in attack decisions, select and dispense munitions and report results .

These unmanned systems will augment manned platform in every facet of operations on the ground, see, air and space, including the information dominance and manipulation [21]. On a lighter vein it may be presumed that future catastrophe might occur by merely uttering "Alexa - "Drop a Bomb".

Blue Eyes Intelligence Engineering \& Sciences Publication 


\section{FINDINGS AND RECOMMENDATIONS}

Noel Sharkey, professor of IA and robotics at University of Sheffield and chair of International committee for Robots Arms Control mentions " after all the hype about face recognition technologies it turns out that they work really badly for women of darker shades of skin There are many adversarial tests showing these technologies can be gamed or misled.

[22]" The application of AI for handling COVID 19 has been a mixed bag of success and failures till now. AI could not create swarms of Doctors who would search the patient for quarantine/ treatment. It has been experienced that all the AI applications in medical health have been incredibly successful but in very narrow range of applications. For example a medical diagnosis app excellent in diagnosing heart problem might diagnose a patient of cancer/ TB with some rare kind of heart problem. Pl refer the results of Survey attached as Appendix. The survey brings out the role of human which need to override AI machine for decision making thus defeat the very purpose of reliance on AI. To reign the development of AI on correct lines few recommendations are as follows :-

7.1 At National level at present no law governs AI arms race in world it is free for all; US is running Drone Swarm experiment, Russia intends to make a battalion of killer robots and no one knows what China is up to. Hence there is a possibility of converting an AI machine into an effective and indiscriminate mass killing device. Therefore in order to avoid next lockdown situation there is an urgent need to hold an international convention on this aspect. All countries must formulate a National Strategy for development of AI. Regulations are to be formulated for ethical, safe, secure and privacy assured usage of $\mathrm{Al}$ in defence.

7.2 At Military level we need to decide on preferred technology and path for procurement with defined phases for Al adoption to be a part of defence strategy and war fighting.

7.3 Preparation of Standard Operating Procedures (SOPs) for the development and delivery of Al projects and formulate policy for IP Rights. AI algorithms should be robust against manipulation. The criteria of responsibility, transparency, auditability, incorruptibility, predictability and a tendency to not make innocent victims scream with helpless frustration are equally applicable to AI machines [23]. There is a need to evolve and adopt standards for technology development and delivery process for $\mathrm{Al}$ projects and lay down the prioritisation of $\mathrm{Al}$ use at system and subsystem levels.

7.4 We need to review the scaling and adoption plan of Al-led and Al-enabled systems and processes with the user groups. All Governments need to lay down budget allocation separately for emerging technologies.

7.5 AI still being a new and ever expanding field a general lack of AI fluency is there even in the technical community in most of the countries. This Lack of collaboration between the industry and academia needs to be fulfilled.

7.6 We need to attract top talent and incentivise it by apt recognition. During recruitment of personals, we tend to identify people who are good at operating machines and vehicles rather than computers. We should :-

7.6.1 Identify the Right Candidates - Based on services requirements we need to identify the right candidates and sponsor them for long-term courses leading to Diploma, Master and PhD degrees.

7.6.2 Higher Domain Knowledge - Defence personnel should be encouraged to enrol or work at leading $\mathrm{Al}$ technology companies and academic institutes to rapidly gain Al knowledge.

\section{CONCLUSION}

Commoditisation and weaponisation of AI is evident across the board. If an intelligent automation system can save the price of both financial and human cost the investment is justified. AI has the capability to predict future attacks or crisis situation and other forms of information. This knowledge has to be leveraged further in building current and future talent for human needs. While the progress of AI is rapid we need to understand that simply because technology allows for the successful development of LAWS it does not mean that we should. To avoid any mishap the human in the loop and strong global conventions would be required. Instead of feeding data to AI machines which may be misused by AI machines in future the policy of starving data might be a defensive alternative.

\section{APPENDIX FINDINGS OF SURVEY}
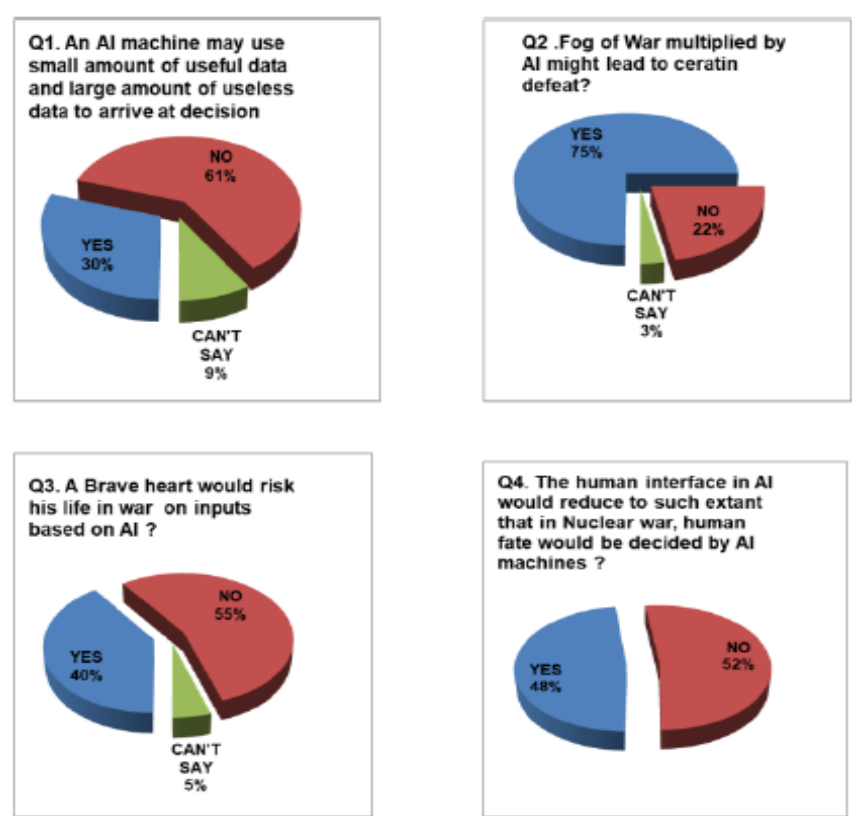

Q4. The human interface in Al would reduce to such extant that in Nuclear war, human by $A$ fate would be d
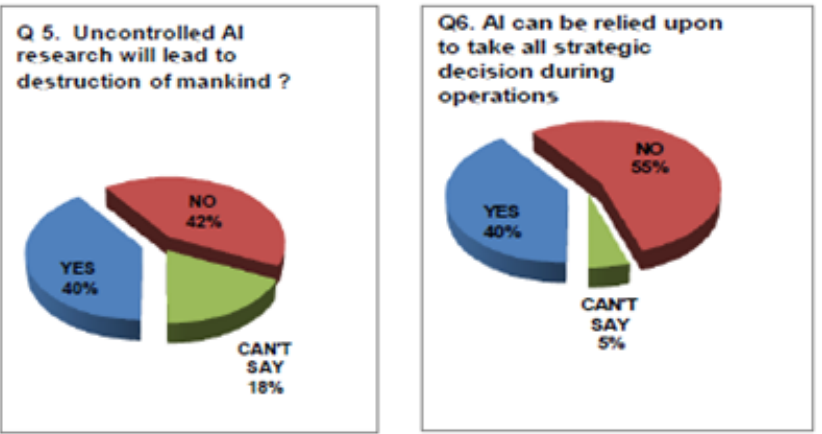

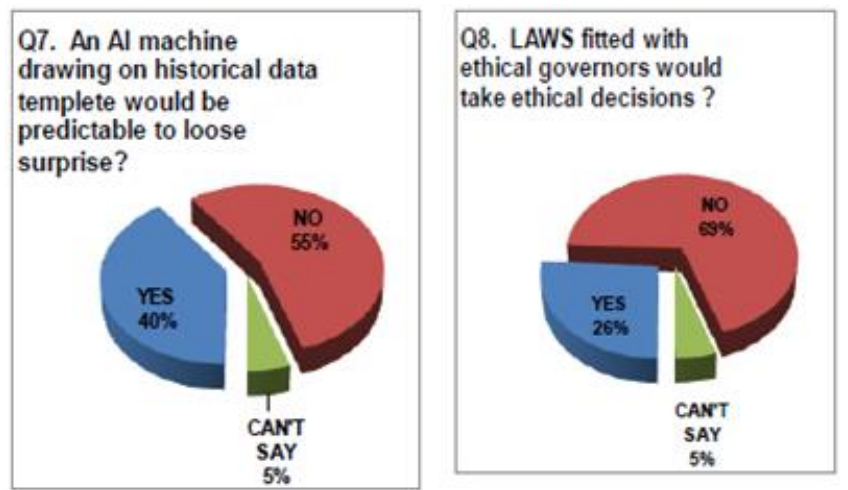

\section{REFERENCES}

1. Kenneth Payne (2018) Artificial Intelligence: A Revolution in Strategic

Affairs?Survival, https://www.tandfonline.com/doi/abs/10.1080/0039 6338.2018.1518374, Published in International Institute for Strategic Studies, accessed on 10 Mar 20.

2. Peter Layton (2018), Algorithmic Warfare Applying Artificial Intelligence to Warfighting, Available at isbn 978192562267 https://www.researchgate.net/ publication, accessed on 12 Mar 20.

3. COVID 19 and Artificial intelligence : Protecting healthcare workers and curbing the spread News item published by Elsevier ltd available onhttps://www.sciencedirect.com/journal/the-lancet-digital-health/vol /2/issue/4, accessed on 10 May 20.

4. Raju Vaishya(2020) et. Al. in article Artificial intelligence (AI) applications for COVID 19 Pandemic available at https://doi.org/10.1016/j.dsx.2020.04.012 diabetes India Published by Elsevier Ltd, accessed on 11 May 20.

5. Artificial Intelligence to Predict Coronavirus Spread available at https://www.cnbc.com/2020/03/03/bluedot-used-artificial-intelligence -to-predict-coronavirus-spread.html, accessed on 14 Mar 20.

6. https://www.alibabacloud.com/solutions/ct-image-analytics, accessed on 10 Apr 20.

7. Latest AI tools to Fight Coronavirus available at https://analyticsindiamag.com/ can-baidus-latest-ai-tool-fightcoronavirus-linearfold-rna/, accessed on $10 \mathrm{Apr} 20$.

8. Chia Surveillance Technology https://www.nytimes.com/2018/07/08 business/china-surveillance-technology.html , accessed on 20 Mar 20 .

9. COVID 19 and Artificial intelligence, loc. cit.

10. Coker C (2019) AI and the future of war. Scandinavian Journal of Military Studies 2(1), https://sjms.nu/articles/10.31374/sjms.26/ accessed on 10 Apr 20.

11. Michael T Clare and Tom Dispatch, Artificial Intelligence and future of Warfare by available on "https://truthout.org/articles/artificialintelligence -and-the-future-of-war/", accessed on 10 Mar 20.

12. Project Maven to deploy Computer Algorithms available at https://dailydefensenews.wordpress.com/2017/07/21/project-maven-t o-deploy-computer-algorithms-to-war-zone-by-years-end//2 accessed on 20 Mar 20.

13. https://www.firstpost.com/tech/news-analysis/scientists-develop-ai-sy stems-that-can-identify-people-by-analysing-how-they-walk-4484943 html, accessed on 10 Mar20.

14. Artificial Intelligence help reign in Pandemic available at https://www.livemint.com/opinion/columns/can-artificial-intelligence -help-rein-in-pandemics-it-seems-that-it-can-11588269161927.html accessed on 13 Mar 20.

15. Michael T Clare and Tom Dispatch, loc. cit. ibid. Peter Layton, loc.cit.

16. ML Cummings (2017) Research paper "Artificial Intelligence and Future of Warfare" published by Chatham House, The Royal Institute of International Affairs available at https://www.chathamhouse.org/ publication/artificial-intelligence-and-future-warfare accessed on 10 Apr 20.

17. Five of the Scariest Prediction for AI, available at https://www.cnbc.com/2018/08/01/five-of-the-scariest-predictions-for -ai.html accessed on 30Mar 20.

18. Panwar RS (2019), Artificial Intelligence in Military Operations: Technology and Ethics Indian Perspective available at https://usiofindia.org/publication/usi-journal/artificial-intelligence-inmilitary-operations-technology-and-ethics-indian-perspective/ accessed on 25 Apr 20.

19. US Army Training and Doctrine Command, "Concept of Employment for unmanned Systems (Draft)" 24 Aug 1999 P4, available at https://www.thefreelibrary.com/Future+warfare+and+the+decline+of +human+decisionmaking.-a0290734209 See also US Army Training and Doctrine Command, Unmanned System Future capability (draft) Aug 1999.

20. https://www.popularmechanics.com/military/research/a23133118/us-a i-robots-warfare/, accessed on 12 Mar 20

21. Bostrom Nick and Eliezer Yudkowsky (2011) The Ethics of Artificial Intelligence in Cambridge handbook of Artificial Intelligence published by New York Cambridge University Press available at https://nickbostrom.com/ethics/artificial-intelligence.pdf, accessed on 20 Apr 20.

\section{AUTHORS PROFILE}

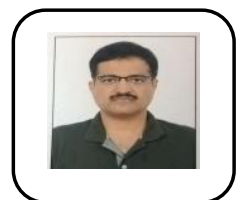

Mr. Naveen Rathi, is Post graduate of Weapon Technology and done PGDM in Finance. He is an avid writer and also presented papers during various International Conference. His Paper on Artificail Intelligence was presented during Interantional Seminar on 'Embracing the Transformative Times' at JPIIT , NOIDA on 07 Feb 20.Another paper written by him on 'Make in India - Challenges in Defense Sector' was published in IJMRA Volume 9 Issue 5(2) of May 2019. Another paper on similar subject found place in MILIT Journal (ISSN 2278-6872), Vol VIII, Dec 2019 of IAT Pune. He participated in seminar on 'COVID-19 and Emotional Wellness' organised by the School of Education, Central University of Jammu and presented paper on Artificail Intelligence in International seminar conducted by St Martins Engineering College, Scunderabad on 17 Jun 20.

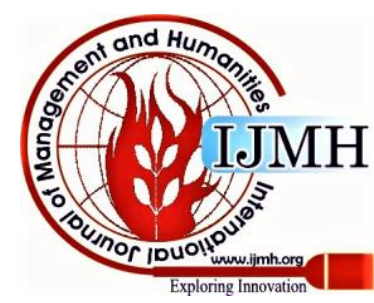

